\title{
Public Awareness of the Benefits of Aspirin Use in the Management of Acute Myocardial Infarction
}

\author{
Gilat L. Grunau ${ }^{*}$, , Pamela A. Ratner ${ }^{1,2}$, Paul M. Galdas ${ }^{1,2}$ and Shahadut Hossain ${ }^{1}$ \\ ${ }^{l}$ NEXUS \& ${ }^{2}$ School of Nursing, University of British Columbia, Vancouver, Canada
}

\begin{abstract}
Background: Aspirin is known to be an effective agent in the tertiary prevention of acute myocardial infarction (AMI). The current recommendation is that aspirin should be given immediately during an AMI and continued afterward. Despite the recognized benefits of aspirin therapy, only a small portion of individuals take aspirin when experiencing acute chest pain. The objective of this study was to determine the proportion of the public that knew that aspirin administration during an AMI is beneficial and to examine factors associated with such knowledge.

Methods: A telephone survey was completed by 976 people $40+$ years of age, in Metro Vancouver, Canada.

Results: Only $33.7 \%$ of the sample indicated that they were 'very likely' or 'definitely likely' to take an aspirin if they were experiencing symptoms of a "heart attack." In multivariate analysis, older individuals, better educated individuals, and those with AMI experience in self, family member or friend, were more likely to report that they would take an aspirin. Chinese immigrants, compared with Canadian-born participants, were less likely to indicate that they would take an aspirin.

Conclusions: Interventions for increasing awareness of the benefits of aspirin during an AMI should be made a priority in general, and with a particular focus on the Chinese-Canadian community.
\end{abstract}

Keywords: Myocardial infarction, aspirin, ethnic groups.

\section{BACKGROUND}

Aspirin is known to be an effective agent in the tertiary prevention of acute myocardial infarction (AMI) [1], and can prevent many premature deaths during AMI $[2,3]$. A large randomized trial (the Second International Study of Infarct Survival (ISIS-2)) [4], examined the effect of aspirin in individuals admitted to hospitals up to 24 hours after the onset of suspected AMI. Three treatment groups (with 17, 187 patients) were compared against placebo: (a) 1-hour intravenous infusion of streptokinase, (b) 1 month of entericcoated aspirin, (c) both active treatments, and (d) neither active treatment (placebo). At 5-weeks follow up, each active treatment alone resulted in a significant reduction in mortality. Moreover, the combination of both active treatments was shown to have a significantly larger effect in reducing mortality than either treatment alone. What is more, the results of the study demonstrated that aspirin does not significantly increase the incidence of cerebral hemorrhage or bleeds requiring transfusion.(4) A 10-year follow up of this sample showed that the benefits of aspirin continued in the long term [5].

The current recommendation is that for all patients with an AMI, aspirin should be given immediately and continued afterward [2]. It is important to encourage patients to use aspirin when experiencing symptoms of AMI because the earlier the aspirin is taken the sooner it will result in treatment benefits [6].

*Address correspondence to this author at the GENESIS and NEXUS, University of British Columbia, 302-6190 Agronomy Road, Vancouver, BC V6T 1Z3, Canada; Tel: +001 604-822-5045; Fax: +001 604-822-7869; E-mail: gilatgrunau@gmail.com
The Heart and Stroke Foundation of Canada's website recommends that individuals should chew and swallow a 325 $\mathrm{mg}$ aspirin tablet or two $80 \mathrm{mg}$ tablets if they are experiencing symptoms of an AMI, in addition to calling emergency services [7]. Similar recommendations are found on the American Heart Association (AHA) and the British Heart Foundation websites $[8,9]$.

Despite the recognized benefits of aspirin therapy, researchers have found that only a small portion of individuals who call emergency services when experiencing acute chest pain have concomitantly taken aspirin. Jaffy et al. examined data from 694 individuals who called emergency services because they were experiencing chest pain and found that only $15 \%$ had taken aspirin before the arrival of emergency personnel [10].

The purpose of this study was to determine the proportion of the public, in a large Canadian urban center, that knew that aspirin administration was recommended at the onset of symptoms suggestive of AMI. We also examined factors associated with such knowledge and whether individuals who had a history of AMI were more aware of the benefits of aspirin administration during AMI. This study was part of a larger investigation of sociodemographic, clinical, cognitive, psychological, and social factors associated with treatment seeking for cardiac symptoms.

\section{METHODS}

\section{Sample, Data Collection, and Questionnaire}

This study used data collected from a telephone survey which was conducted as part of a larger study which is 
described in detail elsewhere $[11,12]$. A random sample of men and women $(\mathrm{n}=3,419), 40$ years of age and older, living in Metro Vancouver, Canada, was obtained. To ensure representation of the Chinese and South Asian communities (the two largest immigrant communities in Metro Vancouver) [13], the questionnaire was administered in four languages (English, Punjabi, Mandarin, and Cantonese).

The British Columbia (BC) Ministry of Health Services, Client Registry Database served as the sampling frame. This database includes people who have applied for the Medical Services Plan (public health insurance plan) and who have lived in the province for at least 3 months. It includes the name, date of birth, gender, address, and telephone number of all enrollees [14]. The BC Ministry of Health's Confidentiality Agreement: Security Provisions for Personal Information in Individual Identifiable Form and the University of British Columbia's Behavioural Research Ethics Board gave approval for accessing the database and conducting the study.

A letter of introduction, in English, with an explanation of how the potential participant's contact information was obtained, an informed consent form, a pre-addressed stamped envelope and the researchers' contact information were mailed to all randomly selected persons. People with names that suggested they might be of Chinese or South Asian origin also received a translated copy in the appropriate language. A reminder telephone call was made to those who did not return signed consent forms. Once consent was received, up to 15 telephone calls were made day and evening, 7 days a week, to either administer the questionnaire or to establish a suitable time for the telephone interview $[15,16]$.

The questionnaire included 71 questions, one of which was the focus of this study: "How likely is it that you would take an aspirin if you were experiencing symptoms of a heart attack? Not at all likely? Somewhat likely? Very likely? Definitely?" Other items considered in this study were: history of AMI, either personally, in the family or among friends, ethnicity/immigration status, gender, education, and income.

To be classified as "Chinese" the participants had to meet the following criteria: (a) they were born in a country other than Canada and (b) they self-identified as Chinese or spoke Chinese as their first language. Other participants were classified as "South Asian" if they: (a) were born in a country other than Canada and (b) self-identified as Punjabi or spoke Punjabi or Hindi as their first language.

The rest of the respondents who were immigrants to Canada were classified as being "not born in Canada" (and not Chinese or South Asian). Three respondents were born in Canada, identified as Chinese, and functioned entirely in English, although they spoke Chinese as their first language. This very small number of unique respondents was excluded from the analyses.

\section{ANALYSIS}

Data analyses were conducted with SPSS (SPSS, Chicago, IL 2006) and S-Plus (Insightful Corp, Seattle, WA 2001). Nineteen percent of the participants did not disclose their household income. To avoid the potential bias associated with deletion of these respondents, multiple imputation of values for missing data was used $[17,18]$. Five complete sets of data were imputed using the outcome variable, the covariates, and other variables predictive of those covariates.

Multiple imputation were done under the conditional Gaussian model, which handles missing data when some of the variables are factors and some are numeric [19]. The model assumes that the cell frequencies of the contingency table formed by the factor variables follow a multinomial distribution and, conditional on the factor variables, the numerical variables follow a multivariate normal distribution. Thus the model appropriately handles the distributional assumptions for categorical and numerical variables. Finally, the missing values were imputed by a data augmentation (DA) algorithm, which is a chain based imputation algorithm. To consolidate the analysis, an appropriate pooling method was used to combine the point and interval estimates. For the point estimates, the average of the estimated coefficients across the five datasets was calculated. For the interval estimates, the standard errors (SEs) of the pooled estimates were computed by the method suggested by Rubin [20], which accounts for both the within imputation and between imputation variability. The confidence intervals were then computed based on the pooled SEs.

The responses to the question regarding aspirin use were re-coded with "not at all likely" and "somewhat likely" coded as "no" and the other two categories coded as "yes" (16 individuals responded "don't know" and were excluded from the multivariate analysis).

Univariate analysis was conducted using the Chi-square test. Multivariate logistic regression analysis was used to examine the relationships between the predictors and the outcome variable. Predictor variables in the regression model included: gender, age, ethnicity/immigration status, income, education, and history of acute myocardial infarction (AMI), either personally, in the family or among friends. All continuous and ordinal variables in the multivariable models were assessed for conformity to a linear gradient. Multicollinearity and goodness of fit were appraised in the models. A $p$ value of $<0.05$ was defined as statistically significant.

\section{RESULTS}

About 28\% (976) of the 3, 419 names from the client registry completed the survey, and $22.2 \%$ (759) refused to participate. The remaining $49.2 \%(1,684)$ people could not be contacted or were ineligible ("wrong" or fax number (39\%), number not in service (26\%), could not be contacted $(25 \%)$, no longer lived in the sampling area $(5 \%)$, spoke a language other than those offered (4\%), or had died (1\%)). A conservatively estimated response rate was $28.5 \%$ (the number of completed interviews divided by the number of completed interviews, the number of refusals, and all cases of unknown eligibility) [21]. A more liberally estimated response rate was $56.3 \%$ (the number of completed interviews divided by the number of completed interviews and the refusals; this eliminated those who had died, did not meet the eligibility criteria, and for whom eligibility could not be determined). 
The mean age of the sample was 55.5 years (SD 11.1) and $43.5 \%$ were men. About one half of the participants (55.1\%) were born in Canada, $22.7 \%$ were not born in Canada (and were not identified as Chinese or South Asian), $15.3 \%$ were Chinese (not born in Canada), and $6.9 \%$ were South Asian (not born in Canada). For a detailed description of the sample see Ratner et al. [11, 12], which present analyses of factors associated with the participants' ability to recognize symptoms of AMI and likelihood of enlisting emergency medical services.

\section{Univariate Analysis}

Of the entire sample, $33.7 \%$ said they were 'very likely' or 'definitely likely' to take an aspirin if they were experiencing symptoms of a heart attack. The responses were not significantly associated with education or gender. The likelihood of taking an aspirin was found to be statistically significantly associated with ethnicity/immigration status. Only $20.8 \%$ of the Chinese-Canadian respondents reported that they would take an aspirin in the event that they experienced symptoms of a heart attack (compared with $36.9 \%$ of those born in Canada) (OR $=0.45 ; 95 \%$ CI: 0.29 , 0.69) (see Table 1).
The likelihood of taking an aspirin was found to be significantly associated with the variable "AMI in oneself, a family member or a friend." Individuals who had had an AMI or had a family member or friend who had had an AMI were more likely to report that they would take an aspirin $(\mathrm{OR}=1.64 ; 95 \%$ CI: $1.21,2.24)$ (see Table 2$)$.

\section{Multivariate Analysis}

Age, education, AMI (in self, family member or friend) and ethnicity/immigration status were significant variables in the multivariate analysis, with older individuals, better educated individuals, and those with AMI (in self, family or friend) being more likely to report that they would take an aspirin. After controlling for gender, age, income, education, and AMI (in self, family or friend), the Chinese-Canadian participants were significantly less likely to respond that they would take an aspirin. Interactions between gender and ethnicity/immigration status, gender and AMI (in self, family member or friend), and AMI (in self, family member or friend) and ethnicity/immigration status were tested and were not statistically significant (see Table $\mathbf{3}$ ).

Table 1. Likelihood of Taking Aspirin at Onset of Acute Myocardial Infarction by Ethnicity/Immigration Status

\begin{tabular}{|c|c|c|c|c|c|}
\hline & $\begin{array}{c}\text { Born in } \\
\text { Canada } n(\%)\end{array}$ & $\begin{array}{l}\text { Not Born in Canada } \\
\text { (Not Chinese/South Asian) n (\%) }\end{array}$ & $\begin{array}{l}\text { South Asian } \\
\text { n (\%) }\end{array}$ & $\begin{array}{c}\text { Chinese } \\
\text { n (\%) }\end{array}$ & $\begin{array}{l}\text { Total } \\
\text { n (\%) }\end{array}$ \\
\hline \multicolumn{6}{|c|}{ Would take an aspirin if experiencing symptoms of a heart attack ${ }^{\phi^{*}}$} \\
\hline Yes & $198(36.9)$ & $76(34.4)$ & $22(32.8)$ & $31(20.8)$ & $327(33.6)$ \\
\hline Don't know & $2(0.4)$ & $3(1.4)$ & $5(7.5)$ & $6(4.0)$ & $16(1.6)$ \\
\hline
\end{tabular}

"The original response options for the question were: "not at all likely, " "somewhat likely, " "very likely, " or "definitely;" these were recoded as a dichotomous variable with "not at all likely" and "somewhat likely" coded as "no" and the other two categories coded as "yes."

${ }^{*}$ Chi-square $=36.53, \mathrm{df}=6, p<0.001$.

Only $5.2 \%$ participants $(\mathrm{n}=51)$ had a history of AMI. The likelihood of taking an aspirin was not associated with a history of AMI (OR $=0.90 ; 95 \%$ CI $0.49,1.65)$. Most of the individuals (64.7\%) who had had an AMI answered that they were "not at all likely" or "somewhat likely" to take an aspirin if they were experiencing symptoms of a heart attack. Identical results were found for those who did not have an AMI history.

\section{DISCUSSION}

The findings presented here are of concern in light of clinical practice recommendations about the benefits of aspirin administration soon after the onset of AMI [8, 22]. Approximately $65 \%$ of the individuals in our study were "not at all likely" or "somewhat likely" to take aspirin if experiencing symptoms suggestive of AMI. This coheres with the low utilization of aspirin during AMI identified elsewhere [10].

Table 2. Likelihood of Taking Aspirin at Onset of Acute Myocardial Infarction by AMI History (in Self, Family Member, or Friend)

\begin{tabular}{|c|c|c|c|}
\hline & $\begin{array}{l}\text { No AMI in Self, Family or Friend } \\
\text { n (\%) }\end{array}$ & $\begin{array}{l}\text { Yes, AMI in Self, Family or Friend } \\
\text { n (\%) }\end{array}$ & Total \\
\hline \multicolumn{4}{|c|}{ Would take an aspirin if experiencing symptoms of a heart attack $*$} \\
\hline Yes & $72(26.3)$ & $256(36.9)$ & $328(33.9)$ \\
\hline Don't know & $5(1.8)$ & $11(1.6)$ & $16(1.7)$ \\
\hline
\end{tabular}


Table 3. Logistic Regression Model for Predictors of Likelihood of Taking Aspirin at Onset of Acute Myocardial Infarction (n = 943)

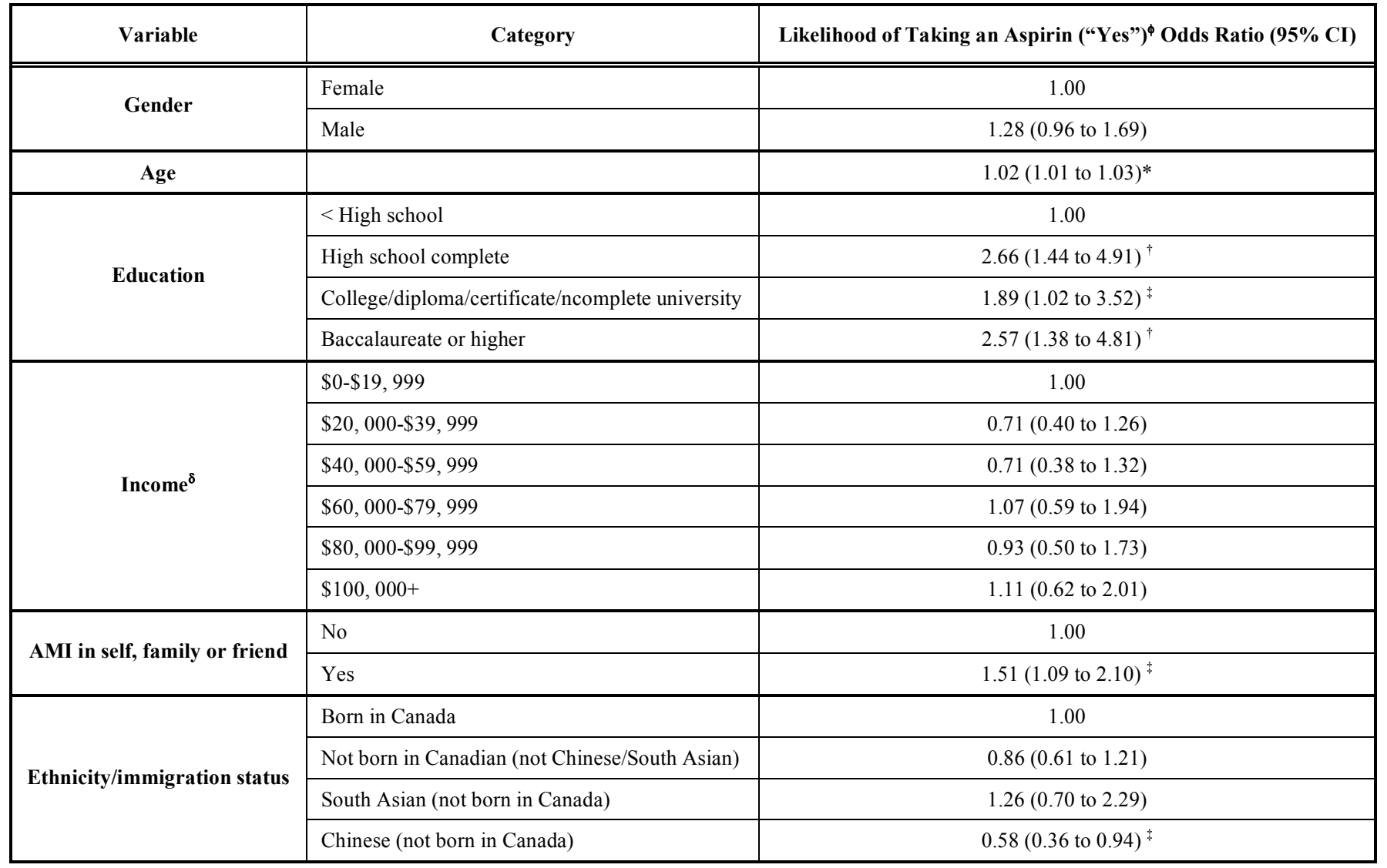

${ }^{*} p<0.001$.

${ }^{\dagger} p<0.01$.

${ }^{\ddagger} p<0.05$.

"Responses to the question regarding likelihood of taking an aspirin were recoded in the following method: those who responded "not at all likely" or "somewhat likely" were coded as "no" and those who responded "very likely" or "definitely" were coded as 'yes'. 'Don't know' responses were coded as missing - there were 16 responses in this category.

${ }^{\delta}$ Multiple imputations of values for missing data for income were used. A set of covariates and other variables predictive of those covariates were used to impute five complete sets of data. Logistic regression analysis was completed for each dataset and the estimated coefficients were averaged across the five datasets.

We found no differences in the potential administration of aspirin between those who had a history of AMI and those who did not. Surprisingly, this indicates that individuals with a history of AMI have not been educated about the benefits of aspirin in tertiary prevention. We expected that individuals with a history of AMI would be more aware of the benefits of aspirin use during AMI and therefore would be more likely to report that they would take aspirin. One possible explanation for our finding is that there was a lack of statistical power to detect this difference, if one exists in the population, considering that only $5.2 \%$ (51 individuals) had a history of AMI. However, when we examined the group of individuals who had had an AMI or angina (93 individuals; $9.5 \%$ of the sample), we still found no association.

Whereas some individuals with a history of AMI could already be taking aspirin long-term, and would derive no benefit from more aspirin, there is concern about poor compliance in this population. For example, a study investigating the impact of depression on adherence to aspirin use after acute coronary syndrome found that $10.5 \%$ of non-depressed patients, $9.8 \%$ of remittent depressed patients, and $42.1 \%$ of persistently depressed patients were poor adherents (using a cutoff of $\leq 75 \%$ of the time as an indication of non-adherence) [23]. Thus, we cannot assume that all post-AMI patients routinely take aspirin.

The likelihood of taking aspirin was associated with an AMI history in one's self, a family member, or a friend; individuals who had such exposure were more likely to report that they would take aspirin. However, a large percentage of these individuals $(61.5 \%)$ responded that they were "not at all likely" or "somewhat likely" to take aspirin, which is of concern.

Our multivariate analysis results showed that after controlling for important variables such as age, income, education, and AMI in self, family member or friend, the Chinese-Canadian participants were least likely to indicate that they would take an aspirin when experiencing symptoms of an AMI. This finding is consistent with other results from this survey reported elsewhere [12], which indicate that the Chinese-Canadian community is ill-informed about the risks and management of cardiovascular disease in general. In another study of Chinese communities in the Canadian cities of Vancouver and Toronto, similar lack of awareness of heart disease was found [24]. Our study adds to this knowledge by showing that there is a lack of awareness specifically regarding the benefits of aspirin use during a 
heart attack. The Canadian-South-Asian group included only 67 individuals, thus it is possible that there was lack of power to detect any differences in this group.

Knowledge of the benefits of aspirin use has been shown to be associated with actual aspirin use [25]. Previous interventions aimed at increasing individuals' knowledge of the benefits of aspirin have been shown to have positive outcomes. For instance, Meischke et al. conducted a randomized trial to evaluate whether a kit delivered by firefighters (which included a list of warning signs of AMI, recommendations to call 9-1-1 and to take an aspirin for chest pain (325 mg of uncoated adult aspirin), and a space to record medications/allergies and important phone numbers) helped seniors become better prepared to deal with the symptoms of AMI [6]. The researchers found that remembering that a kit had been delivered was significantly associated with aspirin administration during an AMI.

The true response rate for this study was between $28.5 \%$ and $56.3 \%$, which is typical for surveys of this kind. When comparing our sample to the Vancouver population (based on the 2001 Census [26], we found that it was sufficiently representative with regard to ethnic classification (i.e., $15.3 \%$ individuals classified as Chinese in our sample compared with $16.6 \%$ found in the Census; $6.9 \%$ classified as South Asian in our sample compared with $6.2 \%$ found in the Census). However, our sample included too few individuals with reported household income of less than $\$ 20,000$ and with less than high school education; these are people often underrepresented in surveys.

Another limitation is that the questionnaire was administered over the telephone, which may have led to different results than those conducted through face-to-face interviews. However, some studies, such as the one conducted by Marcus and Crane have found that results are favorable for telephone surveys [27]. A major concern with telephone surveys is under-coverage of individuals who are less likely to have a telephone; usually low-income minorities and people with lower education levels. However, as described above, our sample is representative of the ethnic distribution of the Metro Vancouver population. We used multiple imputation to minimize the bias that may have resulted from missing data for family income, which is often a limitation of telephone surveys [27].

Although ethnic differences have been found elsewhere, there has yet to be a study that has explored intervention outcomes in a sample of Chinese-Canadian individuals [28]. When planning interventions for Chinese-Canadians, it will be important to take into consideration the barriers to health services and health promotion faced by this population. Researchers have investigated various issues of concern for Chinese immigrants to Canada [29], the USA, and Australia [30]. The chief barriers to access to health services faced by Chinese Canadians, found in a study by Lai et al. [29], were related to linguistic, cultural, and ethnic differences between immigrants and their service providers. Being able to speak in Mandarin or Cantonese, with their health care providers, was noted to be very important to Chinese Australians [30]. The authors recommended that the involvement of bilingual health workers would likely make participation in health promotion more attractive to this population.
Other researchers also have recommended heart disease prevention programs for the Chinese immigrant population, especially aimed at increasing fruit and vegetable consumption and regular physical activity [31, 32]. Our investigation suggests that in addition to such interventions, there should be a focus on aspirin utilization. Additional studies examining methods to increase aspirin usage, at the onset of AMI symptoms, are required, especially with the Chinese-Canadian population.

\section{ACKNOWLEDGEMENTS}

This research was supported by a grant (MOP-53065) from the Canadian Institutes of Health Research and infrastructure support from the Michael Smith Foundation for Health Research (MSFHR). Dr. Grunau acknowledges GENESIS for post-doctoral training support. Dr. Ratner holds a MSFHR Senior Scholar award. Dr. Hossain holds a MSFHR postdoctoral award.

Institution at which work originated: University of British Columbia.

\section{REFERENCES}

[1] Hennekens CH. Update on aspirin in the treatment and prevention of cardiovascular disease. Am J Manag Care 2002; 8: S691-700.

[2] Hennekens $\mathrm{CH}$. Aspirin in the treatment and prevention of cardiovascular disease: current perspectives and future directions. Curr Atheroscler Rep 2007; 9: 409-16.

[3] Hennekens CH, Schneider WR. The need for wider and appropriate utilization of aspirin and statins in the treatment and prevention of cardiovascular disease. Expert Rev Cardiovasc Ther 2008; 6: 95107.

[4] Randomised trial of intravenous streptokinase, oral aspirin, both, or neither among 17, 187 cases of suspected acute myocardial infarction: ISIS-2. ISIS-2 (Second International Study of Infarct Survival) Collaborative Group. Lancet 1988; 2: 349-60.

[5] Baigent C, Collins R, Appleby P, Parish S, Sleight P, Peto R. ISIS2: 10 year survival among patients with suspected acute myocardial infarction in randomised comparison of intravenous streptokinase, oral aspirin, both, or neither. The ISIS-2 (Second International Study of Infarct Survival) Collaborative Group. BMJ 1998; 316: 1337-43.

[6] Meischke H, Diehr P, Rowe S, Cagle A, Eisenberg M. Evaluation of a public education program delivered by firefighters on early recognition of a heart attack. Eval Health Prof 2004; 27: 3-21.

[7] Heart and Stroke Foundation of Canada. Heart attack warning signals. Available from: http://www.heartandstroke.com/site/c.ikI QLcMWJtE/b.3483917/k.8E6D/Warning_signals.htm. [cited 22 May 2008].

[8] American Heart Association. Aspirin in heart attack and stroke prevention. Available from: http://www.americanheart.org/pres enter.jhtml?identifier=4456 [cited 22 May 2008].

[9] British Heart Foundation. Angina. Available from: http://www.bhf. org.uk/living_with_a_heart_con dition/understanding_heart_conditions/ types_of_heart_conditions/angina.aspx [cited 25 Jan 2008].

[10] Jaffy $\overline{M B}$, Meischke H, Eisenberg MS. Prevalence of aspirin use among patients calling 9-1-1 for chest pain. Acad Emerg Med 1998; 5: 1146-9.

[11] Ratner PA, Johnson JL, Mackay M, Tu AW, Hossain S. Knowledge of "heart attack" symptoms in a Canadian urban community. Clin Med Cardiol 2008; 2: 201-13.

[12] Ratner PA, Tzianetas R, Tu AW, et al. Myocardial infarction symptom recognition by the lay public: the role of gender and ethnicity. J Epidemiol Commun Health 2006; 60: 606-15.

[13] Statistics Canada. Community profiles 2001 Census of Canada. Ottawa: Statistics Canada 2004.

[14] Krenten-Boaretto B, Buxton J, Dore K, Fyfe M, Middleton D, McEwen S. Using provincial client registries for selection of control subjects: lessons learned. Can Commun Dis Rep 2003; 29: 173-9.

[15] Salant P, Dillman D. How to conduct your own survey. New York: John Wiley 1994. 
[16] Frey J, Oishi S. How to conduct interviews by telephone and in person. Thousand Oaks, CA: SAGE 1995.

[17] Schafer JL, Olsen MK. Multiple imputation for multivariate missing-data problems: a data analyst's perspective. Multivariate Behav Res 1998; 33: 545-71.

[18] Schimert J, Schafer JL, Hesterberg T, Fraley C, Clarkson DB. Analyzing data with missing values in S-PLUS2001: Available from: http://www.msi.co.jp/ splus/support/download/missing.pdf

[19] Schafer JL. Analysis of incomplete multivariate data. London: Chapman and Hall 1997.

[20] Rubin DB. Multiple imputation for nonresponse in surveys. New York: John Wiley \& Sons 1987.

[21] Mosca L, Linfante AH, Benjamin EJ, et al. National study of physician awareness and adherence to cardiovascular disease prevention guidelines. Circulation 2005; 111: 499-510.

[22] Heart and Stroke Foundation of Canada. Role of ASA in secondary prevention. Available from: http://www.heartandstroke.com/ site/c.ikIQLcMWJtE/b.3734831/k.5EF7/Role of ASA in seconda ry_prevention.htm [cited 22 May 2008].

[23] Rieckmann N, Kronish IM, Haas D, et al. Persistent depressive symptoms lower aspirin adherence after acute coronary syndromes. Am Heart J 2006; 152: 922-7.

[24] Chow CM, Chu JY, Tu JV, Moe GW. Lack of awareness of heart disease and stroke among Chinese Canadians: results of a pilot study of the Chinese Canadian Cardiovascular Health Project. Can J Cardiol 2008; 24: 623-8.

[25] Montori VM, Bryant SC, O'Connor AM, Jorgensen NW, Walsh EE, Smith SA. Decisional attributes of patients with diabetes: the aspirin choice. Diabetes Care 2003; 26: 2804-9.

[26] Statistics Canada. Basic Cross-Tabulations, Census 2001. 2001 Available from: http://www12.statcan.ca/english/census01/products/sta ndard/themes/Index.cfm?Temporal $=2001 \&$ APATH $=3 \&$ FREE $=0$. [cited 23 June 2008].

[27] Marcus AC, Crane LA. Telephone surveys in public health research. Med Care 1986; 24: 97-112.

[28] Brown D, Shepard D, Giles W, Greenlund K, Croft J. Racial differences in the use of aspirin: an important tool for preventing heart disease and stroke. Ethn Dis 2005; 15: 620-6.

[29] Lai DW, Chau SB. Effects of service barriers on health status of older Chinese immigrants in Canada. Soc Work 2007; 52: 261-9.

[30] Kwok C, Sullivan G. Health seeking behaviours among ChineseAustralian women: implications for health promotion programmes. Health (London) 2007; 11: 401-15.

[31] Hislop TG, Teh C, Low A, et al. Knowledge and behaviour regarding heart disease prevention in Chinese Canadian immigrants. Can J Public Health 2008; 99: 232-5.

[32] Taylor VM, Yasui Y, Tu SP, et al. Heart disease prevention among Chinese immigrants. J Commun Health 2007; 32: 299-310.

(c) Grunau et al.; Licensee Bentham Open.

This is an open access article licensed under the terms of the Creative Commons Attribution Non-Commercial License (http://creativecommons.org/licenses/by$\mathrm{nc} / 3.0 /$ ) which permits unrestricted, non-commercial use, distribution and reproduction in any medium, provided the work is properly cited. 